\title{
Retrieval of a Separated Instrument Using Two Different Techniques: Case Reports
}

\author{
${ }^{1}$ Prabhath Singh ${ }^{2}$ Preethee Thomas ${ }^{3}$ Sreeram SR \\ ${ }^{3}$ Vaisakh Nair G \\ 1Head of the Department; Department of Conservative Dentistry \& Endodontics; Amrita School of Dentistry; \\ Amrita Institute of Medical Sciences; Kochi 682041 \\ 2Reader; Department of Conservative Dentistry \& Endodontics; Amrita School of Dentistry; \\ Amrita Institute of Medical Sciences; Kochi 682041 \\ $3 P G$ Conservative Dentistry \& Endodontics; Amrita School of Dentistry; \\ Amrita Institute of Medical Sciences; Kochi 682041
}

\begin{abstract}
Every endodontist may have experienced a variety of emotions associated with instrument separation during root canal treatment. The traditional methods to retrieve such obstructions often require removal of greater amounts of tooth structure, potentially leading to perforation or eventual vertical root fracture. Today, these risks can be minimized with technological advancements in vision, ultrasonics, and microtube retrieval methods. Specifically, the dental operating microscope allows clinicians to visualize most broken instruments and fulfils the age old adage "If you can see it, you can probably do it". This case reports on successful retrieval of separated instruments by two different techniques under magnification.
\end{abstract}

Key Words: Ultrasonic, Instrument Retrieval System, Fractured Instrument.

\section{Introduction}

The separation of instruments during endodontic therapy is a troublesome incident, and its incidence ranges from $2 \%$ to $6 \%$ of the cases investigated ${ }^{1}$. Occasionally during nonsurgical root canal therapy, a separated instrument in a root canal system may block access to the apical terminus. This instrument is usually some type of file or reamer but can include Gates-Glidden or Peeso drills; lentulo spiral paste fillers; thermomechanical gutta-percha compactors; or the tips of hand instruments, such as explorers or gutta-percha spreaders ${ }^{2}$.

The most common causes for file separation are improper use, limitations in physical properties, inadequate access, root canal anatomy, and possibly manufacturing defects ${ }^{2}$.The separated fragment blocks the access to thorough root canal cleaning and shaping procedure apical to the level of separation or irritates the periapex when it juts out of the root apex. This is significant in a tooth, as it affects the final outcome of the endodontic therapy ${ }^{3}$.Hence an attempt to bypass or retrieve the instrument should be made before leaving it and obturating to the level of separation or embarking upon surgery.

The case reports presented here are about the successful retrieval of a separated file tightly wedged in the root canal dentin of a right maxillary second molar, and a left maxillary lateral incisors.

Case 1

\section{Case Reports}

A 45 year old Female Patient reported to the Department of Conservative dentistry \& endodontics of our college, with a chief complaint of dull throbbing pain in the upper right back tooth region since 3 weeks the patient gave a history of previously attempted root canal treatment elsewhere.

On Clinical examination a fractured temporary restoration was present irt $17 \&$ tooth was tender to percussion. Radiographic examination revealed a fractured instrument in the distobuccal root of maxillary right second molar almost to the entire length (Fig 1a). On careful examination we also found a small fragment of headstrom file near the coronal aspect of the fractured instrument. Retrieval was planned using ultrasonic technique under magnification. On the coronal view under magnification we could appreciate the fractured segment in the distobuccal root (Fig 1b) 

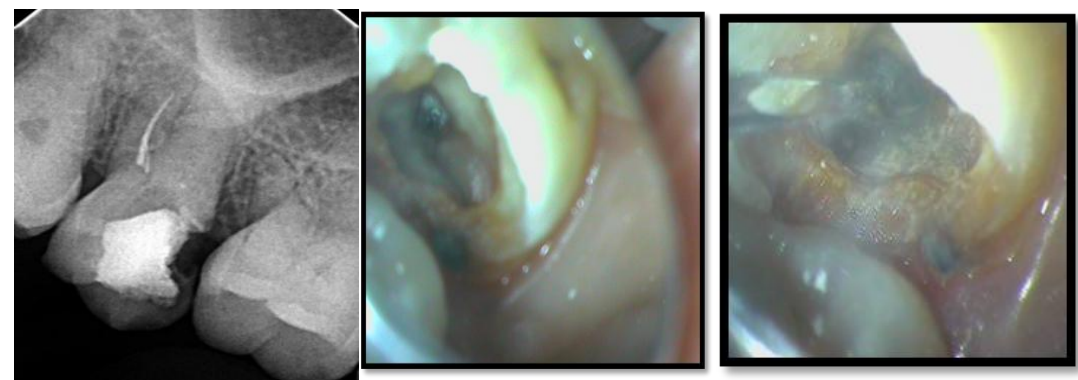

Ultrasonic instrument was used to prepare the radicular dentin around the fragment and the instrument was retrieved successfully (Fig 1c). Cleaning and shaping was completed using hand files, Intra canal medicament was placed and after 1 week the tooth was obturated using lateral condensation technique .Post endodontic permanent restoration was done using composite (Fig1d).
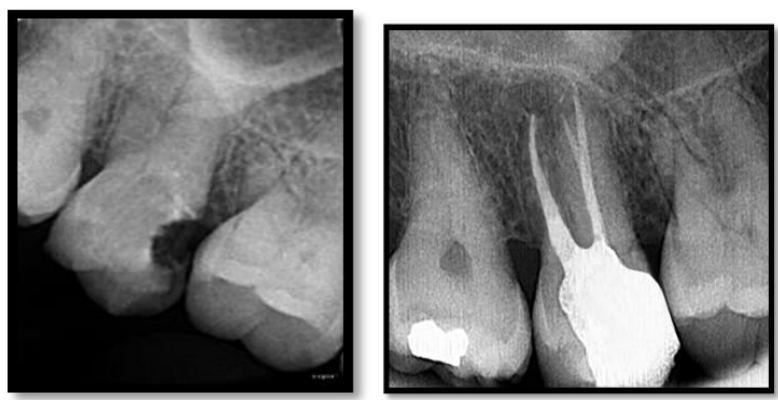

\section{Case 2}

A 29 year old female patient was referred to the department of conservative dentistry and endodontics. On clinical examination there was a discoloured left maxillary lateral incisor with dislodged coronal restoration. IOPAR showed radio opacity till the apex suggestive of fractured instrument (Fig 2a). On magnified coronal view the fractured segment was well appreciated (Fig 2b) Retrieval was planned with a combination of ultrasonics and iRS .

First ultrasonics were used to trephine the radicular dentin in order to expose coronal few millimetres of fractured segment so as to engage iRS (Fig 2c). After engaging to the fractured fragment tightly, the entire assembly was rotated in an anticlockwise direction to unscrew the fragment and was withdrawn (Fig 2d).

After retrieval Cleaning and shaping was done using hand file and a intra canal medicament was placed. After 1 week, obturation was done using lateral condensation technique (Fig 2e), and post endodontic restoration was given.
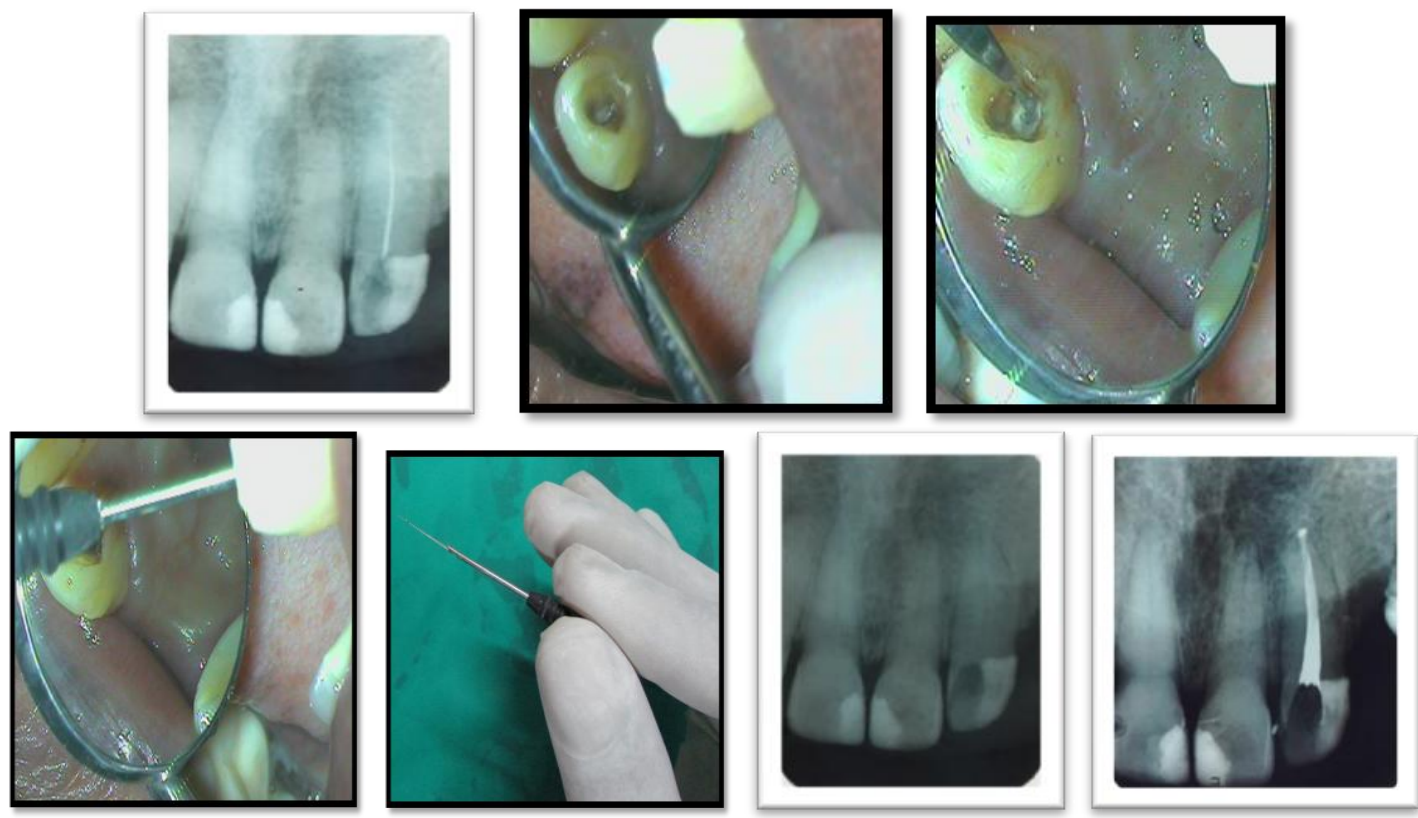


\section{Discussion}

Intracanal separation of instruments usually prevents access to the apex and impedes thorough cleaning and shaping of the root canal, and thus may compromise the outcome of endodontic treatment and reduce the chances of successful retreatment ${ }^{4},{ }^{5}$. In such cases, prognosis following an endodontic therapy depends on the condition of the root canal (vital or nonvital), tooth (symptomatic or asymptomatic, with or without periapical pathology), level of cleaning and shaping at the time of separation, the level of separation in the canal; and is generally lower than that with normal endodontic treatment ${ }^{1}$.

Hence every attempt should be made to bypass or retrieve the separated instrument. The orthograde retrieval depends on cross-sectional diameter, length, curvature of the canal; dentin thickness and morphology of the root; composition, cutting action (clockwise or counter clockwise) of the instrument; length, location, and amount of binding or impaction of the fragment in the canal ${ }^{4}$.

Retrieval was attempted under microscope as the dental operating microscope allows clinicians to visualize most broken instruments and greatly increases the chances for retrieval.Attempting to remove a fragment without adequate visualization highly risks perforation as root curvatures, even though not radiographically visible can easily mislead clinician to remove dentin where it will have little benefit toward file removal ${ }^{6}$.

We used startex no 3 ultrasonic tip for retrieval. Even though manufactures does not recommend these tips for retrieval we chose them due to the limitations in armentarium. Hence from this case report we could say that these can be used in proper combination and skills for retrieval .

However in Case 2 use of these tips alone was not successful we used Startex no 3 tip to Trephines, sands away dentin \& expose coronal few millimetres of fractured segment to Engage iRS

iRS is indicated when ultrasonic efforts prove to be unsuccessful \& may be used to remove broken instruments that are lodged in straight portions of the root or partially around the canal curvature?

\section{Conclusion}

The best antidote for a broken file is prevention. Vast majority of separated instruments can be removed if done with care, time \& right armamentarium, irrespective of the position of the fractured segment

\section{References}

[1]. Arcangelo CM, Varvara G, Fazio PD. Broken instrument removal two cases. J Endod 2000;26:568-70.

[2]. Roda RS, Gettleman BH. Nonsurgical retreatment. In: Cohen S, Burns RC, editors. Pathways of the pulp, 9th ed. St. Louis: CV Mosby; 2006. p. 982-90.

[3]. Pai AR, Kamath MP, Basnet P. Retrieval of a separated file using Masserann technique: A case report. Kathmandu Univ Med J 2006;4:238-42.

[4]. Hulsmann M. Methods for removing metal obstruction from theroot canal. Endod Dent Traumatol 1993;9:223-37.

[5]. Okiji T. Modified usage of the Masserann Kit for removingintracanal broken instruments. J Endod 2003;29:466-7.

[6]. Ruddle CJ: Microendodontic nonsurgical retreatment, in Microscopes in Endodontics, Dent Clin of North Am 41:3, pp. 429-454, W.B. Saunders, Philadelphia, July 1997.

[7]. Roda RS, Gettleman BH. Nonsurgical retreatment. In: Cohen S, Burns RC, editors. Pathways of the pulp, 9th ed. St. Louis: CV Mosby; 2006. p. 982-90. 